\title{
Paranasal Sinus Carcinoma
}

National Cancer Institute

\section{Source}

National Cancer Institute. Paranasal Sinus Carcinoma. NCI Thesaurus. Code C6014.

A malignant epithelial neoplasm arising in the paranasal sinus. 